\title{
A NOTE ON ZERO-DIMENSIONAL SPACES WITH THE STAR-FINITE PROPERTY
}

\author{
HANS-CHRISTIAN REICHEL
}

\begin{abstract}
The paper provides necessary and sufficient conditions for a weakly zero-dimensional metrizable space to be strongly paracompact, i.e., to have the star-finite property. The characterizations use special basis properties of uniformities which induce the topology of $X$, and yield further characteristics of the class of all metric spaces with ind $X=0$ and Ind $X>0$.
\end{abstract}

1. Introduction. A space $X$ is said to have the star-finite property, if every open covering is refined by a star-finite open covering; i.e. any member of the refinement meets at most a finite number of other elements of the refinement. Spaces with the star-finite property are often called strongly paracompact. (As it is known, for locally compact spaces this property coincides with paracompactness.)

In dimension theory the star-finite property plays an important role. P. Roy [Bull. Amer. Math. Soc. 68 (1962), 609-613] has constructed a weakly zero-dimensional metric space $X$, i.e. ind $X=0$, which certainly does not have the star-finite property. The purpose of this paper is to characterize all weakly zero-dimensional metrizable spaces which have the star-finite property. Using results of (among others) B. Banaschewski, B. Fitzpatrick and R. M. Ford, K. Morita, J. de Groot, A. F. Monna and $P$. Nyikos this yields nice characterizations of the class of all metric spaces $X$ with ind $X=0$ and Ind $X>0$.

The conditions of these characterizations involve special basis properties and are outside the realm of dimension theory. At the same time, characterizations of all order-totally-paracompact [2] metrizable spaces with ind $X=0$ are obtained.

2. Preliminaries. A space $X$ has Ind $X=0$ (ind $X=0$ ), if every pair of disjoint closed subsets (points) can be separated by open-closed sets. A. F. Monna and B. Banaschewski have shown that the topology of every space $X$ with ind $X=0$ can be induced by a uniformity $\mathscr{U}$ on $X$ which has a base $\left\{U_{i} \mid i \in I\right\}$ of equivalence relations, i.e. $U_{i} \circ U_{i}=U_{i}, i \in I$ ([4], [1]). (As a defining family of pseudometrics for $\mathscr{U}$ take e.g.

Received by the editors April 13, 1972 and, in revised form, February 10, 1973.

AMS (MOS) subject classifications (1970). Primary 54D20, 54E15; Secondary 54F45. 
$\left\{d_{A} \mid d_{A}(x, y)=1\right.$ iff $x \in A, y \notin A$ or vice versa; moreover $d_{A}(x, y)=0$ in all other cases; $A$ is a clopen subset of $X\}$.)

If such a space has a countable base $\left\{U_{i} \mid i=1,2, \cdots\right\}$ of equivalence relations, there is a nonarchimedean metric $d$ on $X$, which is compatible with $\mathscr{U}$; in other words, $d$ satisfies the strong triangular inequality:

$$
d(x, y) \leqq \max (d(x, z), d(z, y)) \text { for all } x, y, z \in X .
$$

Clearly, if $d(x, z)=\varepsilon, d(z, y)=\eta, \eta \leqq \varepsilon$, then $(x, z) \in U_{\varepsilon} \in \mathscr{U},(z, y) \in$ $U_{\eta} \subset U_{\varepsilon}$. Consequently, $(x, y) \in U_{\varepsilon}^{2}=U_{\varepsilon}$ which is equivalent to $d(x, z) \leqq$ $\max (d(x, z), d(z, y))$.

Now let $X$ be a nonarchimedeanly metrizable space and $B_{\varepsilon}(y)=$ $\{(x, y) \mid d(x, y)<\varepsilon\}$, then the strong triangular inequality implies $B_{1} \subset B_{2}$ or $B_{1} \supset B_{2}$ whenever $B_{1} \cap B_{2} \neq \varnothing$. It follows that any such space $(X, d)$ has the star-finite property [2] and Ind $X=0$ [7]. Conversely, J. de Groot has proved that the topology of any metrizable space $X$ with Ind $X=0$ can be induced by a nonarchimedean metric [3]. (In a quite different setting this theorem has been proved by $F$. Hausdorff before.)

Now we can prove the following theorem.

\section{The results.}

THEOREM 1. A weakly zero-dimensional metrizable space $X$ has the star-finite property if and anly if the topology of $X$ can be induced by $a$ uniform structure which has a totally ordered base (ordered by inclusion) of equivalence relations.

ProOf. By a theorem of K. Morita [5] the star-finite property guarantees ind $X=$ Ind $X$ for every metrizable space $X$. Thus any such metrizable space $X$, ind $X=0$, has Ind $X=0$ and therefore is nonarchimedeanly metrizable. The induced uniform structure $\mathscr{U}$ obviously has a totally ordered base of equivalence relations:

$$
\left\{U_{\varepsilon}=\{(x, y) \mid d(x, y)<\varepsilon\}, \varepsilon>0\right\} .
$$

Conversely, let $\mathscr{B}=\left\{U_{i} \mid i \in I\right\}$ be a totally ordered base of equivalence relations for the uniform space $(X, \mathscr{U})$; then every $U_{i}$ induces a partition of $X$, and, of course, the clopen sets of all these partitions form a base for the topology. Moreover, if two members $A, B$ of this base have nonempty intersection, they belong to different partitions one of which refines the other. So we essentially obtain a base with the following property: two basis sets either have empty intersection or one contains the other. And this implies the star-finite property [2].

REMARK. The second part of the proof goes through without any metrizability condition. We can construct a type of universal space for 
arbitrary uniform spaces $X$ having a totally ordered base $\mathscr{B}$ of equivalence relations: Let $\left\{U_{\sigma} \mid \sigma<\tau\right\}$ be a well-ordered cofinal subset of $\mathscr{B}$, then every $U_{i}$ induces a partition of $X$ into $m_{i}$ clopen sets. Now let $\alpha=\sup m_{i}$ and let $A$ be an arbitrary set of cardinality $\alpha$. For each equivalence class $B_{\sigma}(x), x \in X$, we arbitrarily associate an element of $A$, associating distinct elements with distinct classes. This procedure yields an inverse system of sets $C_{\sigma}$ and we can build the projective limit $C=\operatorname{proj} \lim \left\{C_{\sigma} \mid \sigma<\tau\right\}$ in the category of sets. The points of $C$ can be identified with transfinite sequences $\left(a_{\sigma} \mid \sigma<\tau\right) \in A^{\tau}$. Now for any sequence $\left(a_{\sigma}\right)$ define the system

$$
\left\{B_{\mu}\left(a_{\sigma}\right)=\left\{\left(b_{\sigma}\right) \mid a_{\sigma}=b_{\sigma}, \forall \sigma<\mu\right\}, \mu<\tau\right\}
$$

to be a local base for a topology $t$ on $A^{\tau}$. Thus $C$ has the topology inherited from the space $\left(A^{\tau}, t\right)$ and, obviously, $X$ is homeomorphically imbeddable into $C$.

Spaces $A^{\tau}$ with the topology considered above have been studied to some extent by A. K. Steiner and E. F. Steiner under the name The natural topology on spaces $A^{\tau}$ [J. Math. Anal. Appl. 19 (1967), 174-178].

P. Roy [ $§ 1]$ was the first to construct a metric space $X$ with ind $X=0$ and Ind $X \neq 0$, thus solving the problem of the equivalence of "ind" and "Ind" for metric spaces in the negative. By Theorem 1 we obtain a characterization of all such spaces:

Corollary 2. A metrizable space $X$ has ind $X=0$ and Ind $X>0$ if and only if no uniformity $\mathscr{U}$ compatible with the topology of $X$ has a totally ordered base.

Proof. By Theorem 1 we only have to prove the sufficiency of the condition; but this is obvious by the theorem of Hausdorff-de Groot [§2].

4. Order-totally-paracompact spaces. R. M. Ford in his thesis defined the concept of total paracompactness and showed that ind $X=$ Ind $X$ for totally paracompact metric spaces. Later on, Fitzpatrick and Ford [2] generalized this concept to the concept of order-total-paracompactness which again guarantees ind $X=$ Ind $X$ for metric spaces $X$. Order-totalparacompactness generalizes especially the star-finite property, which is not induced by total paracompactness alone. (Compare also J. A. French in [Duke Math. J. 38 (1971), 251-253].) Let us recall the definitions: $X$ is totally paracompact iff every basis for $X$ has a locally finite subcollection covering $X$. And $X$ is order-totally-paracompact if for any base $G$ there is a linearly ordered collection $(H,<)$ of open sets covering $X$ such that:

(i) every $h \in H$ is contained in an element $g \in G$ such that $B(h) \subset B(g)$, where " $B$ " denotes the boundary of the set. 
(ii) $H$ is initially locally finite, that is: for any $h \in H$ the collection of all $h_{i}<h\left(h_{i} \in H\right)$ is locally finite at each point of $\bar{h}$.

The importance of condition (i) is showed by a result of H. Tamano, Note on paracompactness [J. Math. Kyoto Univ. 3 (1963), 137-143]: a regular space $X$ is paracompact iff for every open cover $G$ of $X$ there is a linearly ordered collection $(H,<)$ of open sets refining $G$ such that $H$ is initially locally finite. Other interesting references in this connection are: $\mathrm{H}$. Tamano and J. E. Vaughan, Paracompactness and elastic spaces [Proc. Amer. Math. Soc. 28 (1971), 299-303]; J. E. Vaughan, Linearly ordered collections and paracompactness [Proc. Amer. Math. Soc. 24 (1970), 186192] and Y. Katuta, $A$ theorem on paracompactness of product spaces [Proc. Japan. Acad. 43 (1967), 614-618].

Now, by Theorem 1, we get the following characterization:

THEOREM 3. For metric spaces $X$ the following properties are equivalent:

(i) ind $X=0$ and $X$ has the star-finite property.

(ii) ind $X=0$ and $X$ is order-totally-paracompact.

(iii) Ind $X=0$.

(iv) The topology of $X$ can be induced by a uniformity $\mathscr{U}$ which has $a$ countable base consisting of equivalence relations $U_{i}$ on $X$.

(v) The topology of $X$ can be induced by a uniformity $\mathscr{U}$ which has a totally ordered base consisting of equivalence relations $U_{i}$ on $X$.

Proof. (i) $\Rightarrow$ (ii) and (ii) $\Rightarrow$ (iii) has been proved by Fitzpatrick and Ford in [2]. (iii) $\Rightarrow$ (iv) follows by the theorem of Hausdorff-de Groot (compare also [1]) and analogously (iv) $\Rightarrow(v)$ because of the metrizability of $X$. The implication $(v) \Rightarrow(i)$, and thus the equivalence of all these properties, is proved in our Theorem 1.

REMARK. The negation of any of these properties gives interesting characterizations of the so-called Roy spaces, i.e. the class of all metric spaces $X$ with ind $X=0$ and Ind $X>0$.

ACKNOWLEDGEMENT. The author would like to thank the referee and P. Nyikos for interesting and worthwhile communications.

\section{REFERENCES}

1. B. Banaschewski, Über nulldimensionale Räume, Math. Nachr. 13 (1955), 129-140. MR 19, 157.

2. B. Fitzpatrick, Jr. and R. M. Ford, On the equivalence of small and large inductive dimension in certain metric spaces, Duke Math. J. 34 (1967), 33-37. MR 34 \#5059.

3. J. de Groot, Non-archimedean metrics in topology, Proc. Amer. Math. Soc. 7 (1956), 948-953. MR 18, 325.

4. A. F. Monna, Remarques sur les métriques non-archimédiennes. I, II, Nederl. Akad. Wetensch. Proc. 53, 470-481=Indag. Math. 12 (1950), 122-133; ibid. 53, 625-635=Indag. Math. 12 (1950), 179-191. MR 12, 41, 1002. 
5. Jun-iti Nagata, Modern dimension theory, Bibliotheca Mathematica. Vol. VI, Interscience, New York, 1965. MR 34 \#8380.

6. P. Nyikos, N-compact spaces, Dissertation, Carnegie-Mellon University, Pittsburgh, Pa., 1972.

7. P. Nyikos and H. C. Reichel, On the structure of zero-dimensional spaces (to appear).

8. H. C. Reichel, On the metrization of the natural topology on the space $A^{B}, \mathrm{~J}$. Math. Anal. Appl. 34 (1971), 644-647. MR 43 \#1116.

9. P. Ucsnay, Über uniforme Räume, Bonn. Math. Schr. 48 (1970), 21-30.

10. A. C. M. van Rooij, Non-archimedean uniformities, Kyungpook Math. J. 10 (1970), 21-30.

Mathematisches Institut, Universität Wien, VienNa, Austria 